\title{
Mosaic 13q14 deletions in peripheral leukocytes of non-hematologic cancer cases and healthy controls
}

\author{
Mitchell J Machiela $^{1}$, Weiyin Zhou ${ }^{1,2}$, Neil Caporaso ${ }^{1}$, Michael Dean ${ }^{1,3}$, Susan M Gapstur ${ }^{4}$, Lynn Goldin ${ }^{1}$, \\ Victoria L Stevens ${ }^{4}$, Meredith Yeager ${ }^{1,2}$ and Stephen J Chanock ${ }^{1}$
}

Loss of $13 q 14.3$ is a chromosomal event found in $~ 50 \%$ of B-cell chronic lymphocytic leukemia (CLL) and monoclonal B-cell lymphocytosis (MBL) cases. Surveys of somatic alterations in solid tumors have shown sporadic 13q14.3 loss in many different tumor types, but not at high frequency in any specific tumor type. In our recent survey of the single-nucleotide polymorphism (SNP) microarray data from 127000 cancer-free or solid tumor cases, we observed mosaic 13q14.3 loss as common autosomal somatic large structural events ( $>2 \mathrm{Mb}$ in size) in blood and buccal-derived DNA. Herein, we examined this region more closely investigating structural mosaic events $<2 \mathrm{Mb}$ using SNP microarray data in 46254 non-hematologic cancer cases and 36229 controls. We detected 60 individuals with 13q14.3 mosaic loss, 1 mosaic copy neutral uniparental disomy and 13 individuals with homozygosity. Although $13 q 14.3$ loss size was variable, the minimally deleted region (MDR) (chr13: 49590 000-49 983 100; GRCh36) was comparable to what is classically reported in MBL and CLL. Breakpoint analysis of the estimated boundaries reveals enrichment for genes and open chromatin. The frequency of 13q14.3 loss significantly increases with increasing age $(P$-value $=0.028)$, but was not significantly different between non-hematological cancer cases and controls $(0.084 \%$ versus $0.058 \% ; P$-value $=0.19)$. These findings suggest that mosaic $13 q 14.3$ losses accumulate with age. Individuals with detected mosaic 13q14.3 deletions may be early, undetected cases of MBL or CLL, but not necessarily all will develop MBL and CLL.

Journal of Human Genetics (2016) 61, 411-418; doi:10.1038/jhg.2015.166; published online 14 January 2016

\section{INTRODUCTION}

Hemizygous and homozygous deletions on the long arm of chromosome 13 are the most common genetic aberrations in B-cell chronic lymphocytic leukemia (CLL) and monoclonal B-cell lymphocytosis (MBL), occurring in $\sim 50 \%$ of cases. ${ }^{1-4}$ The reported deletions are usually large and heterogeneous in size and typically include a minimally deleted region (MDR) on 13q14.3 ( 130 kb). ${ }^{3,5-7}$ The MDR is telomeric to the retinoblastoma gene ( $R B 1)$ and includes DLEU1 and DLEU2, as well as two notable micro-RNAs, MIR15A and MIR16-1. Loss at 13q14 as a single large abnormality can indicate a more favorable CLL clinical course; ${ }^{2}$ however, a higher percentage of cells with 13q14 loss and increased size of 13q14 deletions have been associated with shorter time to treatment. ${ }^{6-11}$ Deletions at $13 \mathrm{q} 14$ are postulated to result in loss of tumor-suppressor activity, but the variable size of the $13 \mathrm{q} 14$ deletion suggests that more than one tumor-suppressor gene is likely present. Further work has shown that downregulation of MIR15A and MIR16-1 in CLL coupled with a CLL-like phenotype in mouse models deficient for the DLEU2/ MIR15A/MIR16-1 cluster indicate that MIR15A and MIR16-1 may be important regulators in CLL pathogenesis. ${ }^{12-14}$
Somatic alterations of $13 \mathrm{q} 14$ have been reported in solid tumors, suggesting a possible role in the carcinogenesis of select non-hematological malignancies. Approximately 6\% of retinoblastoma cases have a $13 \mathrm{q} 14.3$ deletion of the $R B 1$ gene. ${ }^{15,16}$ Sporadic observation of $13 \mathrm{q} 14$ events has been reported in other solid tumors, but not with the consistency observed in CLL or retinoblastoma. For example, pancancer analyses of The Cancer Genome Atlas data show evidence for 13q14 loss in bladder, breast, colon, glioblastoma, head/neck, kidney, lung, ovarian and endometrial tumors. ${ }^{17}$ Allelic loss at $13 \mathrm{q} 14$ has been reported in one-third of prostate tumors. ${ }^{18,19}$ Other studies have reported 13q14 loss with high prostate tumor grade and stage, ${ }^{2}$ as well as increased proliferation and invasiveness of untransformed prostate cells after downregulation of MIR15A and MIR16-1. ${ }^{21}$ Loss of heterozygosity data from breast cancer studies described that a region including 13q12.2-14.3 can be commonly deleted in breast cancer. ${ }^{22}$ Moreover, it has been suggested that $13 \mathrm{q} 14$ allelic imbalance may be associated with breast cancer mortality more than 5 years after diagnosis, ${ }^{23}$ but further studies are needed to confirm this finding. Additional association evidence indicates that ADP-ribosylation factorlike tumor-suppressor gene 1 (ARLTS1, also called ADP-ribosylation

${ }^{1}$ Division of Cancer Epidemiology and Genetics, National Cancer Institute, Rockville, MD, USA; ${ }^{2}$ Cancer Genomics Research Laboratory, Leidos Biomedical Research, Frederick, MD, USA; ${ }^{3}$ Laboratory of Experimental Immunology, Center for Cancer Research, Frederick National Laboratory, Frederick, MD, USA and ${ }^{4}$ Epidemiology Research Program, American Cancer Society, Atlanta, GA, USA

Correspondence: Dr SJ Chanock, Division of Cancer Epidemiology and Genetics, National Cancer Institute, 9609 Medical Center Drive, Gaithersburg, MD 20852, USA.

E-mail: chanocks@mail.nih.gov

Received 21 October 2015; revised 7 December 2015; accepted 10 December 2015; published online 14 January 2016 
factor-like 11 ARL11) located on $13 \mathrm{q} 14.3 \sim 400 \mathrm{~kb}$ centromeric to MIR15A and MIR16-1 may function as a tumor-suppressor gene for lung cancer, ${ }^{24}$ familial breast cancer, ${ }^{25,26}$ melanoma, ${ }^{27}$ ovarian cancer, ${ }^{28}$ prostate cancer ${ }^{29}$ and colorectal cancer. ${ }^{30}$ Overall, the many reports of 13q14 deletions in solid cancers point towards a possible role as a contributing 'driver' event, but further studies are needed to confirm the reported frequencies and, more importantly, establish the functional implications of 13q14 deletion beyond CLL and MBL.

Genetic mosaicism is the coexistence of clonal cellular populations harboring two or more distinct genotypes in an individual. ${ }^{31,32}$ In previous studies of detectable clonal mosaicism, an increased frequency of large structural events detected in a fraction of circulating cells was noted in prediagnostic samples of individuals who later developed a lymphoid malignancy. ${ }^{33,34}$ One of the most common sites for large structural deletions of more than $2 \mathrm{Mb}$ in size included at least $300 \mathrm{~kb}$ of the $13 \mathrm{q} 14.3 \mathrm{MDR}$ region, ${ }^{33,35}$ the region commonly deleted in CLL. In this context, it is notable that mosaic 13q14.3 loss was observed in a fraction of DNA samples collected from individuals diagnosed with solid tumors or who were cancer free. ${ }^{33,35}$ As the screening detection algorithm for single-nucleotide polymorphism (SNP) microarrays conducted as part of cancer genome-wide association study (GWAS) is stable for events $>2 \mathrm{Mb}$ in size, the aim of this analysis was to re-examine our large GWAS data set of more than 80000 individuals to identify additional, smaller 13q14.3 events as well. In addition, we wanted to determine if there is a possible relationship between 13q14.3 events in blood DNA and risk for solid tumors.

\section{MATERIALS AND METHODS \\ Study population}

The Division of Cancer Epidemiology and Genetics and the Cancer Genome Research Laboratory in the NCI have conducted a series of GWASs with commercial SNP microarrays (Illumina Hap300, Hap240, Hap550, Hap610, Hap660, Hap 1, Omni Express, Omni 1, Omni 2.5 and Omni 5). The study set included 82483 participants (46254 non-hematological cancer cases and 36229 cancer-free controls) with blood or buccal DNA, previously known as Total GWAS Set I and II, actually scanned in the Cancer Genome Research Laboratory. ${ }^{35}$ No cases of retinoblastoma were included in either Total GWAS Set I or II. The institutional review board of the participating study centers and the NIH approved the study protocols and informed consent was received for each study participant.

\section{3q14.3 Copy number alteration detection}

B-allele frequency (BAF) and $\log \mathrm{R}$ ratio (LRR) are two metrics used to assess 13 q14.3 mosaic loss in our study population. BAF is a measure of allelic imbalance used to determine stretches of heterozygous SNPs that deviate from the expected value of 0.5 . LRR provides information on copy number in which LRR values $>0$ are evidence for allelic gains, LRR values $<0$ indicate allelic loss and LRR values not deviating from 0 indicate copy neutral events. BAF and LRR values were estimated using methods described previously, ${ }^{36}$ and normalized and corrected using a framework from our previous work. ${ }^{33}$ Based on copy number status, mean heterozygous BAF bands were used to calculate the percentage of cells in the cellular population that contained copy number alterations at 13q14.3. This copy number alteration detection method has been previously validated with additional laboratory techniques for autosomes (e.g., single tandem repeat, multiplex ligation-dependent probe amplification and fluorescent in situ hybridization $)^{37}$ and is described in greater detail in the Methods and Supplementary information of the original analysis. ${ }^{33}$ Stretches of homozygosity were detected as genomic stretches across 13q14.3 with no BAF band for heterozygous SNPs.

Two segmentation algorithms were used to scan genotypes of the nonhematological cancer population for mosaic alterations at the 13q14.3 locus: a LRR-based approach and a BAF-based approach. Both approaches scanned the sample population and surveyed a window around the CLL MDR region on chromosome 13q14.3 between bases 49139793 and 50269706 (GRCh36). The LRR-based approach isolated individuals with evidence of 13q14.3 loss by finding individuals with MDR windows that had mean LRR values significantly less than mean LRR values on chromosome 1 (used as the reference) by applying $t$-tests with unequal variance and a $P$-value $<1.0 \times 10^{-10}$ as the significance threshold. A mean difference of -0.05 was used as a filter to ensure that the statistically significant $13 \mathrm{q} 14.3$ LRR values displayed meaningful differences from the chromosome 1 reference LRR. The BAF-based approach detected individuals with allelic imbalances at 13q14.3 by selecting individuals with MDR windows that had heterozygous $B_{\mathrm{dev}}$ means $\left(B_{\mathrm{dev}}=|\mathrm{BAF}-0.5|\right.$ for heterozygous BAF bands) that significantly differed from $B_{\mathrm{dev}}$ means on chromosome $1\left(P\right.$-value $\left.<1.0 \times 10^{-10}\right)$. A mean $B_{\text {dev }}$ difference of 0.005 was required in statistically significant regions to ensure that meaningful differences in $B_{\mathrm{dev}}$ were detected. Results from the two approaches were merged and manually reviewed to confirm a copy number alteration at the 13q14.3 MDR. Copy number status was assigned and the percentage of cells with altered 13q14.3 nuclei was estimated from heterozygous BAF bands. For individuals with 13q14.3 events that extended beyond the investigated chromosome 13 window, boundaries were adjusted to include the entire 13q event.

\section{Breakpoint analysis}

Mosaic event breakpoint regions were mapped to within $100 \mathrm{~kb}$ from the best estimate of 13q14.3 deletion start and end points using the SNP intensity data. The $100 \mathrm{~kb}$ window was chosen as a conservative estimate of the true breakpoint since start and stop coordinates for events were defined based on the nearest array tagging SNPs, an estimate that is affected by SNP density and the quality of LRR and BAF data around these SNPs. The 60 detected 13q14.3 mosaic deletions resulted in a total of 120 breakpoint regions, roughly $200 \mathrm{~kb}$ in size.

In all, 1000 permutations of 120 randomly selected $200 \mathrm{~kb}$ regions on chromosome 13 were selected to estimate the underlying distribution of chromosome 13 structural elements flanking the 13q14.3 region. To generate these breakpoints, 60 random events were selected from a Gaussian distribution that reflected observed event length $(\mu=10.80 \mathrm{Mb}, \sigma=16.90 \mathrm{Mb})$ and were constrained to overlap the $393 \mathrm{~kb}$ MDR. The first and last Illumina microarray SNPs within simulated events were used to define the estimated breakpoints, and $100 \mathrm{~kb}$ windows were calculated to model the underlying distribution of observed event breakpoints.

University of California Santa Cruz (UCSC) and Encyclopedia of DNA Elements (ENCODE) data tracks were downloaded from the UCSC FTP data portal (ftp://hgdownload.cse.ucsc.edu/) to investigate local DNA characteristics in breakpoint regions as well as in randomly selected regions. Features of interest included gene-rich regions (RefSeq genes, CpG islands), indicators of open chromatin (OH Radical Cleavage Intensity Database (ORChID), DNaseI hypersensitivity (DNaseI HS) peaks, Formaldehyde-Assisted Isolation of Regulatory Elements (FAIRE-Seq) peaks), recombination rate (deCODE sex-averaged) and repetitive elements (short interspersed nuclear elements (SINEs), long interspersed nuclear elements (LINEs), long terminal repeats (LTRs) and segmental duplications). Comparisons between observed breakpoints and random breakpoints were made using mean differences and mean counts of feature elements. Statistical significance was assessed by permutation $P$-value.

\section{Statistical analyses}

Univariate analyses were performed characterizing the relationship of age group $(<50,50-54,55-59,60-64,65-69,70-74$ and $75 \leqslant$ years), gender (male, female), estimated ancestry (\%European, \%African and \%Asian ancestry estimated by SNP genotypes) and non-hematologic cancer (disease-free controls, solid tumor cases overall and substrata of cases by solid tumor type) with 13q14.3 loss. An additional multivariable analysis that included age group, gender, estimated ancestry and non-hematologic cancer, as well as adjustment for contributing study (indicator variables), were carried out using logistic regression. Strata-specific analyses were performed within strata of nonhematological cancer type to investigate whether 13q14.3 deletion was associated with specific non-hematological cancers. 
To investigate differences in frequency between 13q14.3 alterations in our population and CLL prevalence in the US population, age- and sex-specific limited-duration prevalence measures from the November 2011 submission of Surveillance Epidemiology and End Results ${ }^{38}$ data were used based on the US Census data. All statistical analyses were performed in $\mathrm{R}$ version 2.15.1 (R Foundation for Statistical Computing, Vienna, Austria).

\section{RESULTS}

In a combined analysis of 82483 non-hematologic cancer cases and disease-free control DNA drawn from peripheral leukocytes (81\%) and buccal samples (19\%), we detected 60 individuals $(0.073 \%, 95 \%$ confidence interval $(\mathrm{CI})=0.054-0.091 \%)$ with $13 \mathrm{q} 14.3$ mosaic loss between 19.7 and $90.1 \%$. The distribution of events across strata of gender, age group, ancestry and non-hematologic cancer status is displayed in Table 1. Non-hematological cancer cases and controls in our analysis had a frequency of 13q14.3 mosaic loss of 0.084 $(95 \% \mathrm{CI}=0.066-0.115)$ and $0.069(95 \% \mathrm{CI}=0.038-0.089)$ percent, respectively. The majority of the detected $13 \mathrm{q} 14.3$ mosaic losses were in DNA derived from blood; however, three mosaic 13q14.3 deletions were observed in buccal DNA. In addition to the 60 individuals detected with mosaic loss at 13q14.3, our analysis detected one lung cancer case with a copy neutral uniparental disomy that spanned the $13 q 14.3$ region. Stretches of homozygosity within the $13 q 14.3$ region were also observed in 13 individuals, which included 6 controls,

Table 1 Counts and percentages for detected 13q14.3 mosaic loss across population characteristics

\begin{tabular}{|c|c|c|c|c|}
\hline & 13q14.3 Deletion & No 13q14.3 Deletion & Total & P-value \\
\hline \multicolumn{5}{|l|}{ Gender } \\
\hline Male & $40(0.09 \%)$ & 44012 (99.91\%) & $44052(53.41 \%)$ & \\
\hline \multirow[t]{2}{*}{ Female } & $20(0.05 \%)$ & 38411 (99.95\%) & 38431 (46.59\%) & \\
\hline & $60(0.07 \%)$ & 82423 (99.93\%) & 82483 (100.00\%) & 0.0513 \\
\hline \multicolumn{5}{|l|}{ Age group (years) } \\
\hline$<50$ & $2(0.02 \%)$ & 9882 (99.98\%) & $9884(13.12 \%)$ & \\
\hline $50-54$ & $2(0.03 \%)$ & 6437 (99.97\%) & $6439(08.55 \%)$ & \\
\hline $55-59$ & $4(0.04 \%)$ & $9963(99.96 \%)$ & 9967 (13.23\%) & \\
\hline $60-64$ & $8(0.06 \%)$ & 14048 (99.94\%) & $14056(18.65 \%)$ & \\
\hline $65-69$ & $10(0.07 \%)$ & 15174 (99.93\%) & 15184 (20.15\%) & \\
\hline $70-74$ & $13(0.11 \%)$ & 11639 (99.89\%) & 11652 (15.46\%) & \\
\hline \multirow[t]{2}{*}{$\geqslant 75$} & $15(0.18 \%)$ & $8153(99.82 \%)$ & 8168 (10.84\%) & \\
\hline & $54(0.07 \%)$ & 75296 (99.93\%) & $75350(100.00 \%)$ & $0.0018 *$ \\
\hline \multicolumn{5}{|l|}{ Ancestry } \\
\hline$>85 \%$ European & $53(0.10 \%)$ & 54765 (99.90\%) & $54818(72.82 \%)$ & \\
\hline$>85 \%$ African & $0(0.00 \%)$ & 2647 (100.00\%) & 2647 (03.52\%) & \\
\hline \multirow[t]{2}{*}{$>85 \%$ Asian } & $3(0.02 \%)$ & $17810(99.98 \%)$ & $17813(23.66 \%)$ & \\
\hline & $56(0.07 \%)$ & 75222 (99.93\%) & 75278 (100.00\%) & $0.0004^{*}$ \\
\hline \multicolumn{5}{|c|}{ Non-hematologic cancer } \\
\hline Cancer-free & $21(0.06 \%)$ & 36208 (99.94\%) & 36229 (43.92\%) & \\
\hline Cancer & $39(0.08 \%)$ & 46215 (99.92\%) & 46254 (56.08\%) & 0.1932 \\
\hline Bladder & $4(0.08 \%)$ & $5036(99.92 \%)$ & 5040 (10.90\%) & 0.5376 \\
\hline Breast & $2(0.07 \%)$ & $2827(99.93 \%)$ & $2829(06.12 \%)$ & 0.6813 \\
\hline Colorectal & $0(0.00 \%)$ & 95 (100.00\%) & $95(00.21 \%)$ & 1.0000 \\
\hline Endometrial & $6(0.21 \%)$ & 2908 (99.79\%) & $2914(06.30 \%)$ & $0.0129 *$ \\
\hline Esophagus & $0(0.00 \%)$ & 1915 (100.00\%) & 1915 (04.14\%) & 0.6236 \\
\hline Glioma & $0(0.00 \%)$ & 1729 (100.00\%) & 1729 (03.74\%) & 0.6234 \\
\hline Head/neck & $0(0.00 \%)$ & 25 (100.00\%) & $25(00.05 \%)$ & 1.0000 \\
\hline Kidney & $2(0.13 \%)$ & 1589 (99.87\%) & 1591 (03.44\%) & 0.2520 \\
\hline Liver & $0(0.00 \%)$ & $13(100.00 \%)$ & $13(00.03 \%)$ & 1.0000 \\
\hline Lung & $12(0.09 \%)$ & 13251 (99.91\%) & $13263(28.67 \%)$ & 0.2378 \\
\hline Osteosarcoma & $0(0.00 \%)$ & $982(100.00 \%)$ & $982(02.12 \%)$ & 1.0000 \\
\hline Other & $1(1.20 \%)$ & $82(98.80 \%)$ & $83(00.18 \%)$ & 0.0491 * \\
\hline Ovary & $0(0.00 \%)$ & 548 (100.00\%) & $548(01.18 \%)$ & 1.0000 \\
\hline Pancreas & $4(0.10 \%)$ & 3919 (99.90\%) & 3923 (08.48\%) & 0.3002 \\
\hline Prostate & $7(0.10 \%)$ & 7238 (99.90\%) & 7245 (15.66\%) & 0.3051 \\
\hline Skin & $0(0.00 \%)$ & 49 (100.00\%) & $49(00.11 \%)$ & 1.0000 \\
\hline Stomach & $1(0.03 \%)$ & 3360 (99.97\%) & 3361 (07.27\%) & 1.0000 \\
\hline \multirow[t]{2}{*}{ Testis } & $0(0.00 \%)$ & $649(100.00 \%)$ & $649(01.40 \%)$ & 1.0000 \\
\hline & $60(0.07 \%)$ & 82423 (99.93\%) & 82483 (100.00\%) & 0.1932 \\
\hline Total sample & $60(0.07 \%)$ & 82423 (99.93\%) & 82483 (100.00\%) & \\
\hline
\end{tabular}

$P$-values are from Fisher's exact tests with an asterisk $\left(^{*}\right)$ indicating $P<0.05$. For Ancestry, $>85 \%$ European indicates the individual has $>85 \%$ inferred European ancestry. 
2 endometrial cancer cases, 3 lung cancer cases, 1 ovary cancer case and 1 pancreatic cancer case. Individuals with mosaic 13q14.3 deletions showed no preference for developing additional mosaic autosomal events in other chromosomal locations as compared with others with non-13q14.3 mosaic events $(P=0.17)$.

Detected 13q14.3 mosaic losses are plotted in Figure 1 and vary in size and estimated breakpoint locations. The median mosaic loss was $1.9 \mathrm{Mb}$ (interquartile range: $1.1-17.1 \mathrm{Mb}$ ) in size, with the smallest mosaic loss $246 \mathrm{~kb}$ and the largest $>75 \mathrm{Mb}$ in size. Overall, smaller mosaic 13q14 events are more common than larger events (Figure 2a), which parallels what has been seen for large structural mosaic events across the autosomes. ${ }^{35}$ Common breakpoint regions on the centromeric side included clusters at 29.1-32.0, 40.1-43.7 and 49.3-49.6 Mb, whereas breakpoints on the telomeric side primarily clustered around 50.3-52.9 Mb (GRCh36). At a minimum, mosaic 13q14.3 losses detected in peripheral blood or buccal DNA cover a region on chromosome 13 spanning an $\sim 393 \mathrm{~kb}$ region from base pairs 49590000 to 49983100 (GRCh36). This region includes DLEU1 and DLEU2, the ST13P4 pseudogene, and is $\sim 70 \mathrm{~kb}$ telomeric to the location of MIR15A and MIR16-1. The variable size of the detected $13 q 14.3$ mosaic losses resulted in deletions of other known tumorsuppressor genes. When we examined the mapping of the deletions, we observed inclusion of BRCA2 in $10 \%$ of individuals, $R B 1$ in $40 \%$, MIR15A in $87 \%$, MIR16-1 in $87 \%$, DLEU1 in $95 \%$, DLEU2 in $98 \%$ and DLEU7 in $97 \%$.

In an analysis of average LRR values, an indicator of allelic copy number, the majority of individuals with $13 \mathrm{q} 14.3$ mosaic losses affect only one allele (Figure 2a). There was, however, one control individual with evidence for mosaic loss of both alleles at the 13q14.3 locus $(\mathrm{LRR}=-1.58)$. Overall, the observed mosaic proportion, a measurement of the percentage of cells containing 13q14 loss, was between 19.7 and $90.1 \%$, with a mean of $41.4 \%$ (Figure $2 a$ ). As expected, we observed an association between LRR and mosaic proportion in our data, indicating that despite the small size of many 13q14.3 mosaic losses, we were able to calculate highly correlated measures of mosaic percentage $\left(P=8.16 \times 10^{-9}\right.$, adj $\left.R^{2}=0.52\right) \quad$ (Figure $2 \mathrm{~b}$ ). No significant associations were observed between the size of 13 q14.3 deletion and either LRR or mosaic percentage $(P=0.097$ and 0.211 , respectively).

Analysis of the 13q14.3 deletion breakpoints using select bioinformatic data tracks from the UCSC browser and ENCODE indicates substantial clustering of active elements in the region of 13q14.3 loss breakpoints. In comparison with a random sampling of similar-sized regions on other autosomes, we observed a statistically significant enrichment for regions rich in genes and regulatory elements (Figure 3). For instance, RefSeq genes and CpG islands were significantly enriched at $13 \mathrm{q} 14.3$ breakpoints $(P<0.002$; Figure $3 \mathrm{a})$, indicating $13 \mathrm{q} 14.3$ breakpoint regions often occur near genes or promoters of genes. Indicators of open chromatin, ORChID, DNase HS and FAIRE-Seq, were also significantly enriched $(P<0.002$; Figure $3 \mathrm{~b}$ ). There was no evidence indicating an association between recombination rate and location of $13 \mathrm{q} 14.3$ mosaic breakpoints $(P=0.39$; Figure $3 \mathrm{c})$. The distribution of repetitive elements such as SINEs, LINEs, LTRs and segmental duplications suggests clustering in 13q14.3 breakpoint regions (Figure 3d). In particular, SINEs are significantly enriched for $(P<0.002)$, whereas LTRs are less common in these regions $(P=0.002)$. A marginally significant enrichment for segmental duplications was also observed in $13 \mathrm{q} 14.3$ breakpoint regions $(P=0.062)$.

Unadjusted associations with 13q14.3 mosaic deletions were investigated (Table 1). Mosaic loss of 13q14 was slightly more common in males compared with females (0.09 versus 0.05 , respectively, $P=0.051)$. An unadjusted positive association was also observed between mosaic 13q14.3 loss and 5-year age group $(<50,50-54$, $55-59,60-64,65-69,70-74,75+, P=1.8 \times 10^{-3}$ ), ranging from $0.02 \%$ for individuals $<50$ years and increases up to a frequency of $0.18 \%$ for individuals 75 years and older. An unadjusted association with ancestry was also detected $(P$-value $=0.0004)$ with mosaic loss of $13 q 14.3$ most commonly observed in individuals of European ancestry, but less commonly in individuals of African ancestry. Table 1 further illustrates the breakdown of 13q14.3 mosaic events by solid tumor subtype. Overall, no significant difference in 13q14.3 loss was observed between non-hematological cancers $(0.08 \%)$ and controls $(0.06 \%)(P$-value $=0.19)$.

In exploratory multivariable models including study, gender, 5-year age group, ancestry and non-hematologic cancer status, a positive association was only observed for age $(P$-value $=0.028)$. The increased odds for a $13 \mathrm{q} 14.3$ mosaic deletion for 75 years or older compared with those less than age 50 years was 8.70 (95\% CI $=1.65-45.78)$. In the multivariable analysis, there were no associations of overall solid tumor $(P$-value $=0.89)$ or specific tumor subtype with $13 q 14.3$ mosaic loss.

To investigate whether detection of $13 \mathrm{q} 14$ deletion could be detecting early, undiagnosed cases of CLL, age- and gender-specific frequencies of 13q14.3 mosaic loss in our data set were compared with SEER population-based CLL prevalence data (Table 2). Assuming $50 \%$ of all CLL cases have 13q14.3 loss, expected age- and genderstratified counts of $13 \mathrm{q} 14.3$ loss were estimated for our sample set based on SEER limited-duration prevalence data $(0-<34$ years $)$ and US census data. Results indicate that although on average slightly more instances of 13q14.3 mosaic loss are observed among non-hematologic cancer cases and controls, this does not significantly depart from the 13q14.3 losses attributable to undiagnosed CLL that would be expected in a population of this size, age and gender distribution $(P=0.46)$.

\section{DISCUSSION}

Our analysis of 13q14.3 mosaic losses in DNA isolated from blood or buccal cell samples revealed an overall frequency of $0.073 \%$ $(95 \% \mathrm{CI}=0.056-0.094)$ based on detection of $13 \mathrm{q} 14.3$ mosaicism in 60 individuals out of 82483 who were included in non-hematologic cancer GWAS. No significant difference in frequency of $13 \mathrm{q} 14.3$ mosaic loss was detected between non-hematologic cancer cases and controls. We show that the prevalence of mosaic 13q14.3 loss increases with age, a phenomenon observed for all large mosaic events. ${ }^{35,39}$ The size of 13q14.3 mosaic losses can vary, but almost always included an MDR on chromosome 13, from 49590000 to 49983100 (GRCh36). In more than $85 \%$ of instances, mosaic losses included the MIR15A, MIR16-1, DLEU1, DLEU2 and DLEU7 loci. We did not identify a clearly defined set of breakpoints, suggesting that events may not be mapped to specific base pairs but instead to regions of open chromatin.

An enrichment of genes, promoter sites and enhancers around 13q14.3 breakpoints indicates cellular mechanisms related to transcription and gene expression may be important in breakpoint formation. Regions of open chromatin around enhancers and gene-rich regions may also expose more of the DNA backbone to environmental mutagens and could result in higher probabilities for mutational events. The enrichment of SINEs in 13 q14.3 breakpoint regions, as well as a marginally significant enrichment of segmental duplications, suggests that genomic repeat regions could have a role in initiation of events leading 
a

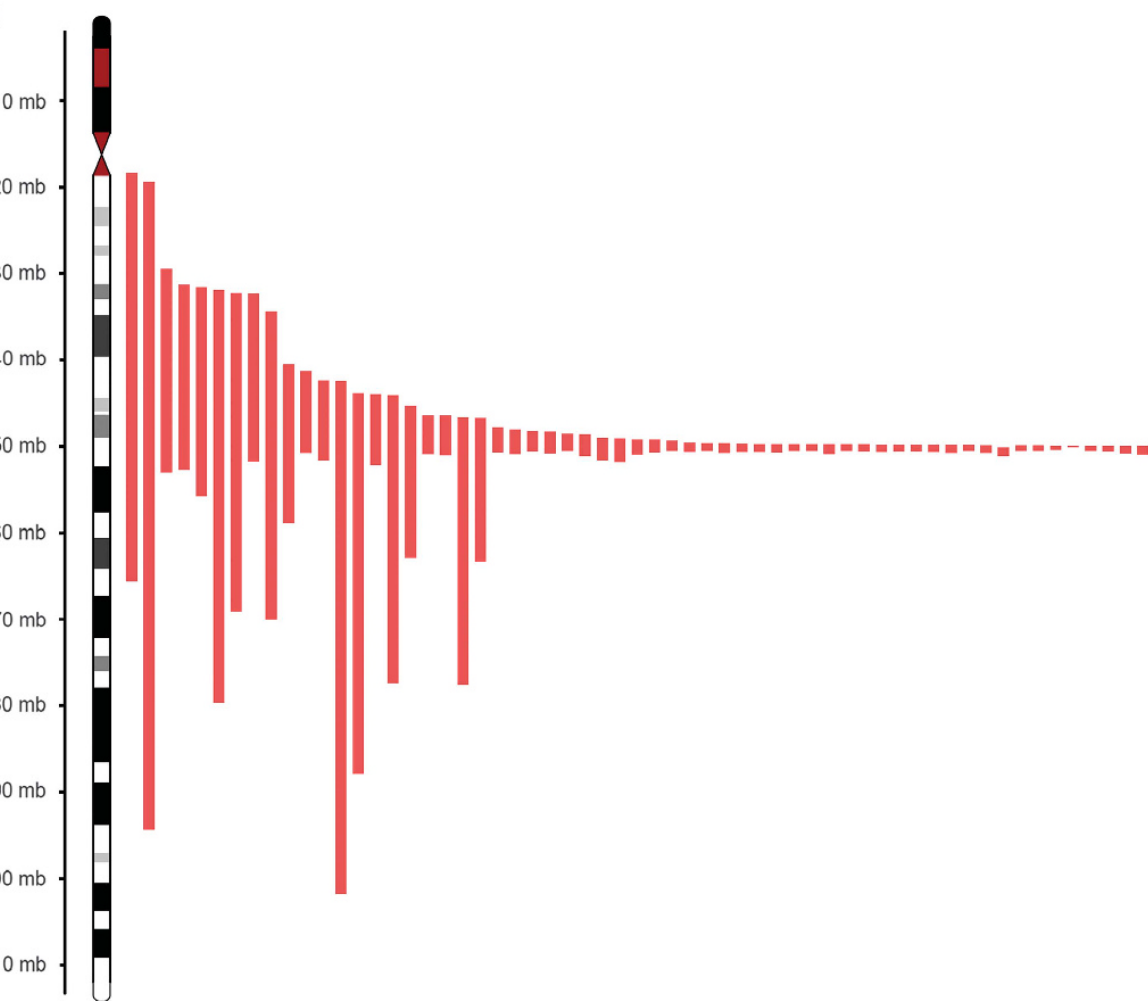

b
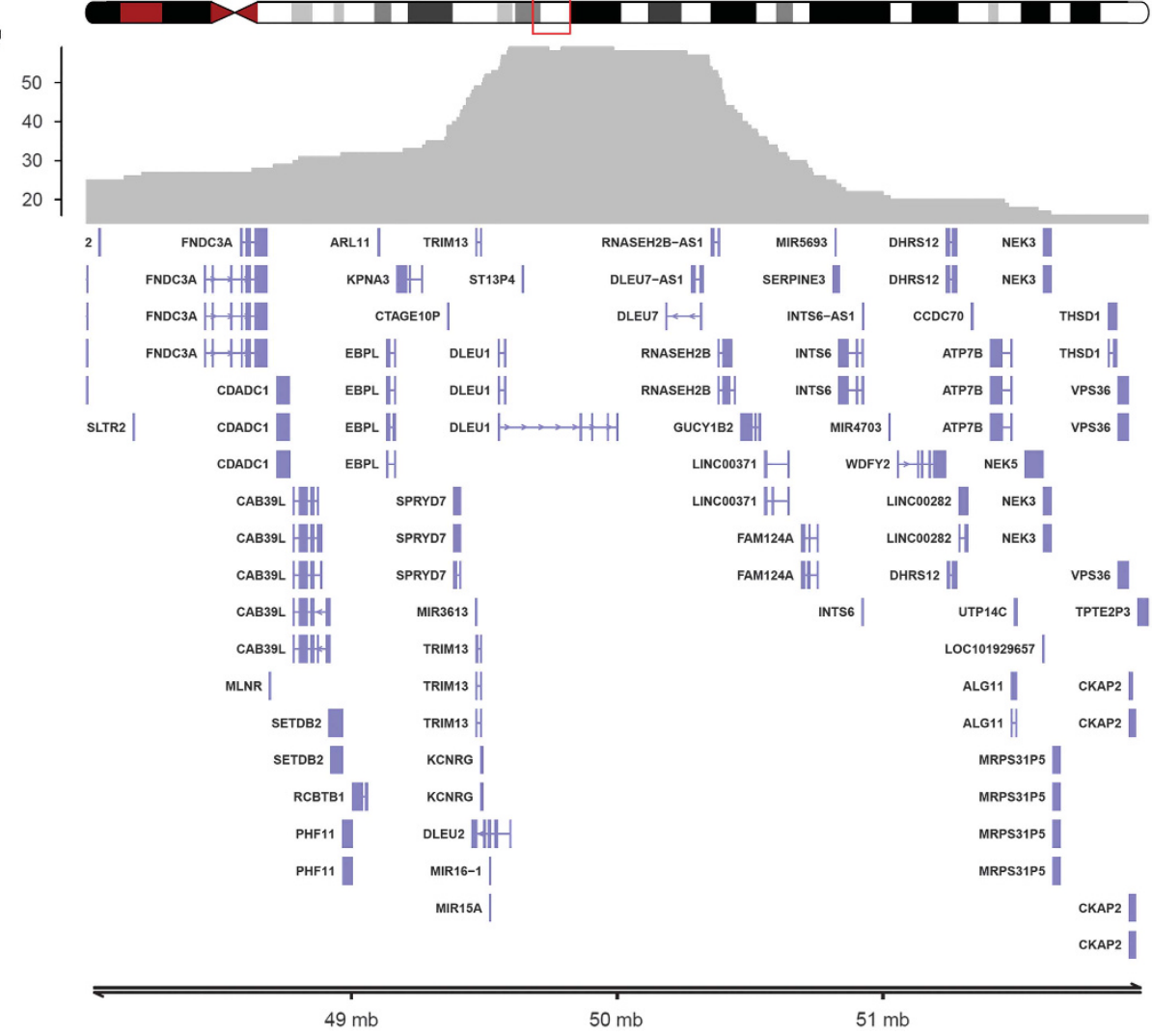

Figure 1 Distribution of 13q14.3 losses. (a) Graphical illustration of the 60 detected mosaic losses that span the minimally deleted region at $13 q 14.3$. (b) Zoom in of the 13q14.3 minimally deleted region showing overlap of detected events (top panel) and genes in the region (bottom panel). 

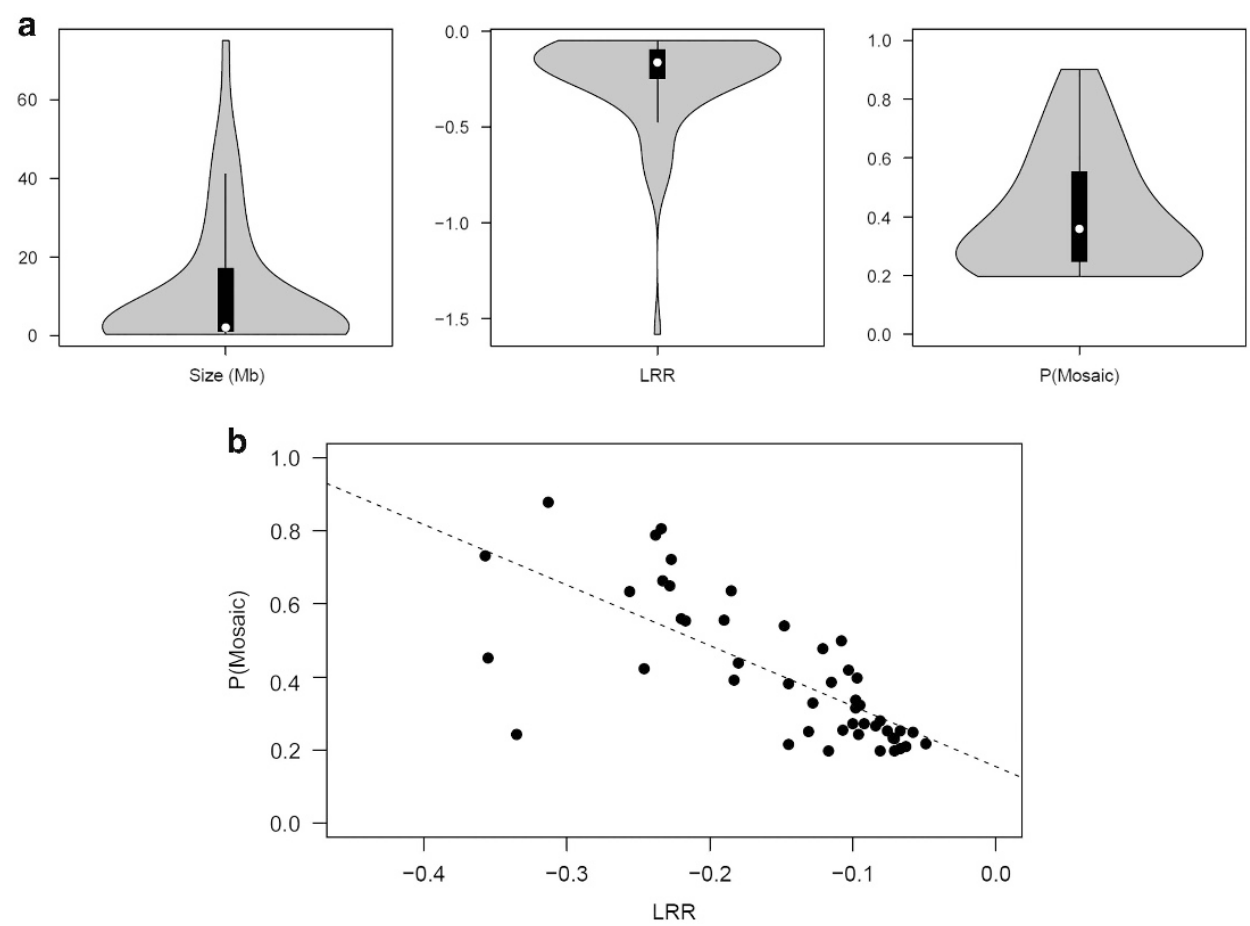

Figure 2 Event size, log $R$ ratio (LRR) and proportion of mosaic cells (P(Mosaic)) for detected 13q14.3 losses. (a) Violin plots of event size, LRR and $P$ (Mosaic) distributions. (b) Relationship between LRR and P(Mosaic) for monoallelic 13q14.3 losses.

a

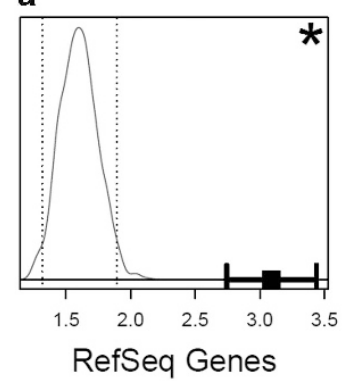

C

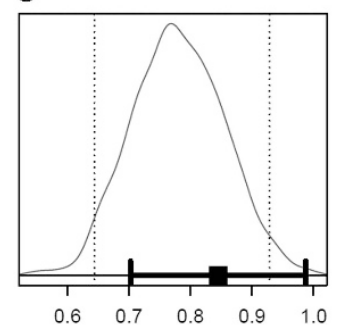

Recombination Rate

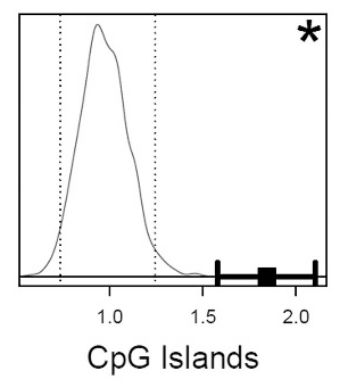

d

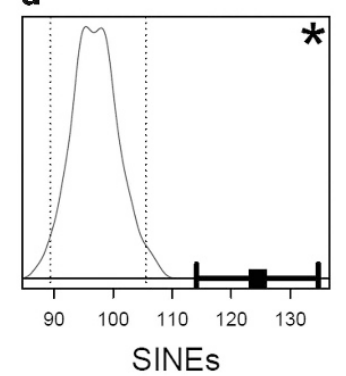

b
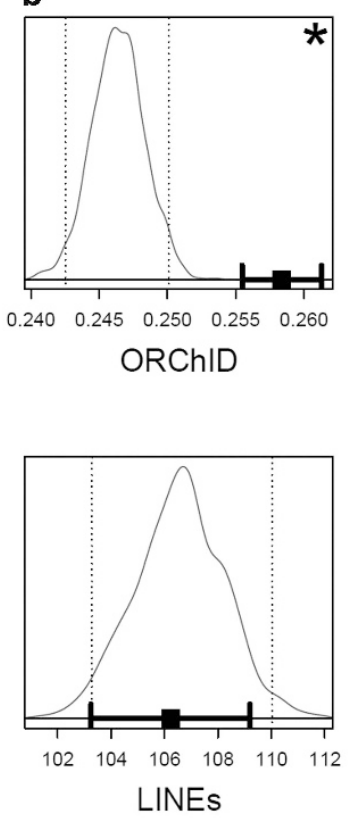

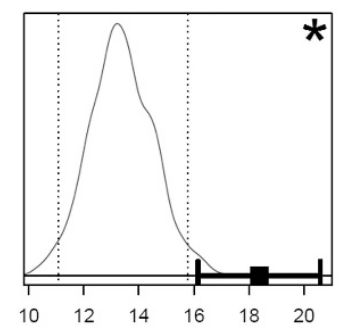

DNasel HS Peaks

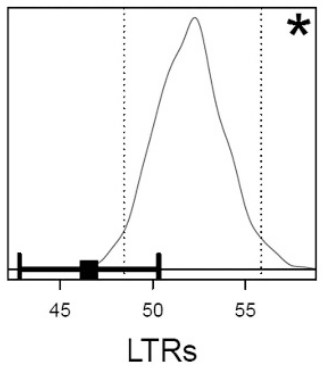

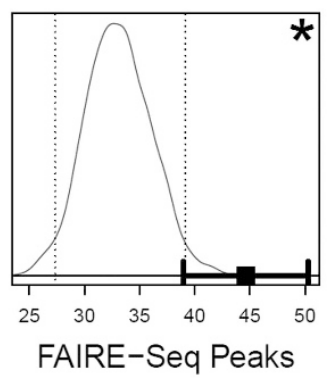

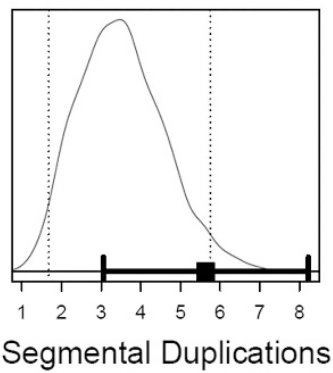

Figure 3 Breakpoint analysis of $13 q 14$ breakpoint regions in comparison with breakpoints from random permutations of similarly sized regions spanning the $13 q 14.3$ minimally deleted region. Features investigated include (a) gene-rich regions, (b) indicators of open chromatin, (c) recombination rate and (d) repetitive elements. Gray distributions are means across 1000 permutations. Black boxes and error bars represent the mean across detected $13 q 14.3$ breakpoints and $95 \%$ confidence interval around the mean. Asterisks $\left({ }^{*}\right)$ indicate $P<0.05$.

to $13 \mathrm{q} 14.3$ mosaic losses. It is less clear what functional role these repetitive elements could contribute to the initiation of somatic 13 q14.3 loss, but transposon activity and mismatch repair may be two important mechanisms to investigate further. Although these findings do not conclusively demonstrate a specific cellular mechanism responsible for the detected 13q14.3 mosaic losses, it does suggest transcription coupled repair, exposure to DNA mutagens and transposon activity may be important mechanisms 
Table 2 Observed versus expected 13q14.3 mosaic deletions

\begin{tabular}{|c|c|c|c|c|c|c|c|}
\hline \multirow[b]{2}{*}{ Sex } & \multirow{2}{*}{$\begin{array}{l}\text { Age group } \\
\text { (years) }\end{array}$} & \multirow[b]{2}{*}{$\mathrm{N}$} & \multicolumn{2}{|c|}{ Observed } & \multicolumn{2}{|c|}{ Expected } & \multirow[b]{2}{*}{ P-value } \\
\hline & & & Count & Percent & Count & Percent & \\
\hline \multirow[t]{5}{*}{ Males } & Ages < $<0$ & 4068 & 1 & $0.025 \%$ & 0.04 & $0.001 \%$ & \\
\hline & Ages $50-64$ & 15959 & 6 & $0.038 \%$ & 4.62 & $0.029 \%$ & \\
\hline & Ages $65-74$ & 16973 & 16 & $0.094 \%$ & 17.50 & $0.103 \%$ & \\
\hline & Ages 75+ & 5225 & 11 & $0.211 \%$ & 9.08 & $0.174 \%$ & \\
\hline & All ages & 42225 & 34 & $0.081 \%$ & 31.24 & $0.074 \%$ & 0.6858 \\
\hline \multirow[t]{6}{*}{ Females } & Ages < $<0$ & 5816 & 1 & $0.017 \%$ & 0.03 & $0.001 \%$ & \\
\hline & Ages 50-64 & 14489 & 6 & $0.041 \%$ & 2.30 & $0.016 \%$ & \\
\hline & Ages $65-74$ & 9831 & 5 & $0.051 \%$ & 5.60 & $0.057 \%$ & \\
\hline & Ages 75+ & 2935 & 2 & $0.068 \%$ & 3.50 & $0.119 \%$ & \\
\hline & All ages & 33071 & 14 & $0.042 \%$ & 11.43 & $0.035 \%$ & 0.5403 \\
\hline & Overall & 75296 & 48 & $0.064 \%$ & 42.67 & $0.057 \%$ & 0.4595 \\
\hline
\end{tabular}

capable of initiating DNA breaks leading to mosaic 13q14.3 deletions.

In an unadjusted analysis, we observed $13 \mathrm{q} 14.3$ mosaic loss were associated with age (5-year age group), continental ancestry and endometrial cancer, but with small numbers these latter observations are most likely false positives. In fact, only the 5-year age group association was observed in the adjusted multivariable analysis. The association between mosaicism and age has been observed in previous studies reporting on overall autosomal mosaicism. ${ }^{33-35,39}$ Notably, 13 q14 deletion events are among the most common large structural somatic events observed in detectable mosaicism. The increasing frequency of $\mathrm{MBL}$ and CLL with age ${ }^{38,40,41}$ makes it challenging to delineate whether detected 13q14.3 mosaic losses are early biomarkers for potential MBL and CLL risk or if such deletions are sentinel events that frequently appear when genomic maintenance capacities begin to deteriorate with age. Additionally, many of the contributing cancer GWASs did not screen for MBL/CLL; thus, there is a substantive possibility that some undiagnosed cases may have existed in our data set.

Our study does not provide sufficient evidence that mosaic 13q14.3 deletions in blood or buccal DNA are significantly associated with solid tumors, despite the literature reports of sporadic observations of 13 q14.3 deletion events in a spectrum of cancers. Publically available The Cancer Genome Atlas data on the cBioPortal suggests that $13 q 14.3$ deletions are present in up to $11 \%$ of prostate tumors, $7 \%$ of bladder tumors, $4 \%$ of endometrial tumors and $3 \%$ of ovary and colorectal tumors. ${ }^{42}$ We surmise that many of these may be passengers and not necessarily drivers of the solid tumors. However, it is important to note that our study on DNA obtained from blood and buccal cells does not provide information about the frequency of $13 q 14.3$ loss in other tissues, which may be a more relevant prognostic feature for tumors that develop in those tissues. In addition, even in our large survey of cancer GWAS, we had limited power to investigate the association between 13q14.3 mosaic loss and solid tumors.

Our study raises an important question regarding the implications of harboring a mosaic 13q14.3 deletion. The most likely possibility is that individuals with mosaic 13q14.3 deletions are instances of early undetected MBL/CLL. The commonly deleted region in our analysis is nearly identical to that seen in MBL/CLL. and the frequency estimate we observe for $13 \mathrm{q} 14.3$ deletions $(0.07 \%)$ is statistically indistinguishable from the expected age and sex prevalence of CLL, assuming that $50 \%$ of CLL cases have a 13q14.3 deletion. Additionally, deletions of $13 \mathrm{q} 14.3$ as the sole abnormality are generally associated with indolent clinical disease ${ }^{2}$ and often appear early in early-stage CLL. ${ }^{43}$ Alternatively, it is plausible that these $13 \mathrm{q} 14.3$ deletions are somatic changes in B- or T-cell populations that serve as sentinel events that frequently appear when genomic maintenance capacities begin to deteriorate with age. The last, and least likely possibility, is that these are inherited events passed from parents to offspring through the germline. The range of allelic proportions for heterozygous SNPs spanning these events (20-90\%) substantially deviates from the $50 \%$ expectation, making this interpretation very unlikely. Further large studies are required to capture a sufficiently large enough set of mosaic 13q14.3 deletions in a prospective manner so as to accurately assess cancer risk.

\section{CONFLICT OF INTEREST}

The authors declare no conflict of interest.

\section{ACKNOWLEDGEMENTS}

This study was funded by the Intramural Research Program of the National Cancer Institute's Division of Cancer Epidemiology and the American Cancer Society.

1 Stilgenbauer, S., Nickolenko, J., Wilhelm, J., Wolf, S., Weitz, S., Döhner, K. et al. Expressed sequences as candidates for a novel tumor suppressor gene at band 13q14 in B-cell chronic lymphocytic leukemia and mantle cell lymphoma. Oncogene 16, 1891-1897 (1998).

2 Döhner, H., Stilgenbauer, S., Benner, A., Leupolt, E., Kröber, A., Bullinger, L. et al. Genomic aberrations and survival in chronic lymphocytic leukemia. N. Engl. J. Med. 343, 1910-1916 (2000)

3 Edelmann, J., Holzmann, K., Miller, F., Winkler, D., Bühler, A., Zenz, T. et al. Highresolution genomic profiling of chronic lymphocytic leukemia reveals new recurrent genomic alterations. Blood 120, 4783-4794 (2012).

4 Rawstron, A., Bennett, F., O'Connor, S., Kwok, M. \& Fenton, J. Monoclonal B-cell lymphocytosis and chronic lymphocytic leukemia. N. Engl. J. Med. 359 575-583 (2008).

5 Mosca, L., Fabris, S., Lionetti, M. \& Todoerti, K. Integrative genomics analyses reveal molecularly distinct subgroups of B-cell chronic lymphocytic leukemia patients with $13 q 14$ deletion. Clin. Cancer Res. 16, 5641-5653 (2010).

6 Ouillette, P., Erba, H., Kujawski, L., Kaminski, M., Shedden, K. \& Malek, S. N. Integrated genomic profiling of chronic lymphocytic leukemia identifies subtypes of deletion 13q14. Cancer Res. 68, 1012-1021 (2008).

7 Ouillette, P., Fossum, S., Parkin, B., Ding, L., Bockenstedt, P., Al-Zoubi, A. et al. Aggressive chronic lymphocytic leukemia with elevated genomic complexity is associated with multiple gene defects in the response to DNA double-strand breaks. Clin. Cancer Res. 16, 835-847 (2010).

8 Hernández, J. A., Rodríguez, A. E., González, M., Benito, R., Fontanillo, C., Sandoval, V. et al. A high number of losses in 13q14 chromosome band is associated with a worse outcome and biological differences in patients with B-cell chronic lymphoid leukemia. Haematologica 94, 364-371 (2009).

9 Van Dyke, D. L., Shanafelt, T. D., Call, T. G., Zent, C. S., Smoley, S. a, Rabe, K. G. et al. A comprehensive evaluation of the prognostic significance of $13 q$ deletions in patients with B-chronic Iymphocytic leukaemia. Br. J. Haematol. 148, 544-550 (2010).

10 Bo, M. D., Rossi, F. M., Rossi, D., Deambrogi, C., Bertoni, F., De Giudice, I. et al. $13 q 14$ Deletion size and number of deleted cells both influence prognosis in chronic lymphocytic leukemia. Genes Chromosomes Cancer 643, 633-643 (2011).

11 Mian, M., Rinaldi, A., Mensah, A., Rossi, D., Ladetto, M., Forconi, F. et al. Del (13q14.3) length matters: an integrated analysis of genomic, fluorescence in situ hybridization and clinical data in 169 chronic lymphocytic leukaemia patients with $13 q$ deletion alone or a normal karyotype. Hematol. Oncol. 53, 46-49 (2012).

12 Calin, G. A., Dumitru, C. D., Shimizu, M., Bichi, R., Zupo, S., Noch, E. et al. Frequent deletions and down-regulation of micro-RNA genes miR15 and miR16 at 13q14 in chronic lymphocytic leukemia. Proc. Natl Acad. Sci. USA 99, 15524-15529 (2002).

13 Klein, U., Lia, M., Crespo, M., Siegel, R., Shen, Q., Mo, T. et al. The DLEU2/miR-15a/ 16-1 cluster controls $B$ cell proliferation and its deletion leads to chronic lymphocytic leukemia. Cancer Cell 17, 28-40 (2010).

14 Lia, M., Carette, A., Tang, H., Shen, Q., Mo, T., Bhagat, G. et al. Functional dissection of the chromosome 13q14 tumor-suppressor locus using transgenic mouse lines. Blood 119, 2981-2990 (2012).

15 Houdayer, C., Gauthier-Villars, M., Lauge, A., Pages-Berhouet, S., Dehainault, C., Caux-Moncoutier, V. et al. Comprehensive screening for constitutional RB1 mutations by DHPLC and QMPSF. Hum. Mutat. 23, 193-202 (2004). 
16 Albrecht, P., Ansperger-Rescher, B., Schuler, A., Zeschnigk, M., Gallie, B. \& Lohmann, D. R. Spectrum of gross deletions and insertions in the RB1 gene in patients with retinoblastoma and association with phenotypic expression. Hum. Mutat. 26, 437-445 (2005)

17 Zack, T. I., Schumacher, S. E., Carter, S. L., Cherniack, A. D., Saksena, G., Tabak, B. et al. Pan-cancer patterns of somatic copy number alteration. Nat. Genet. 45, 1134-1140 (2013).

18 Yin, Z., Spitz, M. R., Babaian, R. J., Strom, S. S., Troncoso, P. \& Kagan, J. Limiting the location of a putative human prostate cancer tumor suppressor gene at chromosome 13q14.3. Oncogene 18, 7576-7583 (1999).

19 Cooney, K. A., Wetzel, J. C., Merajver, S. D., Cooney, A., Macoska, J. A. \& Singleton, P. Distinct regions of allelic loss on $13 q$ in prostate cancer. Cancer Res. 56, 1142-1145 (1996).

20 Dong, J. T., Boyd, J. C. \& Frierson, H. F. Loss of heterozygosity at $13 q 14$ and $13 q 21$ in high grade, high stage prostate cancer. Prostate 49, 166-171 (2001).

21 Bonci, D., Coppola, V., Musumeci, M., Addario, A., Giuffrida, R., Memeo, L. et al. The miR-15a-miR-16-1 cluster controls prostate cancer by targeting multiple oncogenic activities. Nat. Med. 14, 1271-1277 (2008).

22 Osborne, R. \& Hamshere, M. A genome-wide map showing common regions of loss of heterozygosity/allelic imbalance in breast cancer. Cancer Res. 3706-3712 (2000)

23 Voeghtly, L. M., Mamula, K., Campbell, J. L., Shriver, C. D. \& Ellsworth, R. E. Molecular alterations associated with breast cancer mortality. PLOS ONE 7, e46814 (2012).

24 Yendamuri, S., Trapasso, F., Ferracin, M., Cesari, R., Sevignani, C., Shimizu, M. et al. Tumor suppressor functions of ARLTS1 in lung cancers. Cancer Res. 67, 7738-7745 (2007).

25 Akisik, E., Yazici, H. \& Dalay, N. ARLTS1, MDM2 and RAD51 gene variations are associated with familial breast cancer. Mol. Biol. Rep. 38, 343-348 (2011).

26 Frank, B., Hemminki, K., Meindl, A., Wappenschmidt, B., Klaes, R., Schmutzler, R. K. et al. Association of the ARLTS1 Cys148Arg variant with familial breast cancer risk. Int. J. Cancer 118, 2505-2508 (2006).

27 Frank, B., Meyer, P., Boettger, M. B., Hemminki, K., Stapelmann, H., Gast, A. et al. ARLTS1 variants and melanoma risk. Int. J. Cancer 119, 1736-1737 (2006)

28 Petrocca, F., Iliopoulos, D., Qin, H. R., Nicoloso, M. S., Yendamuri, S., Wojcik, S. E. et al. Alterations of the tumor suppressor gene ARLTS1 in ovarian cancer. Cancer Res. 66, 10287-10291 (2006).

29 Siltanen, S., Wahlfors, T., Schindler, M., Saramäki, O. R., Mpindi, J. P., Latonen, L. et al. Contribution of ARLTS1 Cys148Arg (T442C) variant with prostate cancer risk and ARLTS1 function in prostate cancer cells. PLoS One 6, e26595 (2011).
30 Castellví-Bel, S., Castells, A., de Cid, R., Muñoz, J., Balaguer, F., Gonzalo, V. et al. Association of the ARLTS1 Cys148Arg variant with sporadic and familial colorectal cancer. Carcinogenesis 28, 1687-1691 (2007).

31 Strachan, T. \& Read, A. Human Molecular Genetics ( Wiley-Liss, New York, NY, USA, 1999).

32 Machiela, M. J. \& Chanock, S. J. Detectable clonal mosaicism in the human genome. Semin. Hematol. 50, 348-359 (2013).

33 Jacobs, K. B., Yeager, M., Zhou, W., Wacholder, S., Wang, Z., Rodriguez-Santiago, B. et al. Detectable clonal mosaicism and its relationship to aging and cancer. Nat. Genet. 44, 651-658 (2012)

34 Laurie, C. C., Laurie, C. A., Rice, K., Doheny, K. F., Zelnick, L. R., McHugh, C. P. et al. Detectable clonal mosaicism from birth to old age and its relationship to cancer. Nat. Genet. 44, 642-650 (2012).

35 Machiela, M. J., Zhou, W., Sampson, J. N., Dean, M. C., Jacobs, K. B., Black, A. et al. Characterization of large structural genetic mosaicism in human autosomes. Am. J. Hum. Genet. 96, 487-497 (2015).

36 Wang, K. \& Bucan, M. Copy number variation detection via high-density SNP genotyping. CSH Protoc 2008, pdb.top46 (2008).

37 Rodríguez-Santiago, B., Malats, N., Rothman, N., Armengol, L., Garcia-Closas, M., Kogevinas, M. et al. Mosaic uniparental disomies and aneuploidies as large structural variants of the human genome. Am. J. Hum. Genet. 87, 129-138 (2010)

38 Surveillance Research Program. Fast Stats: An interactive tool for access to SEER cancer statistics. National Cancer Institute. http://seer.cancer.gov/faststats. Accessed 23 January 2013.

39 Dumanski, J., Rasi, C., Lönn, M. \& Davies, H. Smoking is associated with mosaic loss of chromosome Y. Science 347, 81-83 (2015).

40 Goldin, L. R., Lanasa, M. C., Slager, S. L., Cerhan, J. R., Vachon, C. M., Strom, S. S. et al. Common occurrence of monoclonal B-cell lymphocytosis among members of highrisk CLL families. Br. J. Haematol. 151, 152-158 (2010).

41 Scarfò, L., Dagklis, A., Scielzo, C., Fazi, C. \& Ghia, P. CLL-like monoclonal B-cell lymphocytosis: are we all bound to have it? Semin. Cancer Biol. 20, 384-390 (2010).

42 Cerami, E., Gao, J., Dogrusoz, U., Gross, B. E., Sumer, S. O., Aksoy, B. A. et al. The cBio cancer genomics portal: an open platform for exploring multidimensional cancer genomics data. Cancer Discov. 2, 401-404 (2012).

43 Shanafelt, T. D., Witzig, T. E., Fink, S. R. Jenkins, R. B. Paternoster, S. F., Smoley, S. A. et al. Prospective evaluation of clonal evolution during long-term follow-up of patients with untreated early-stage chronic lymphocytic leukemia. J. Clin. Oncol. 24, 4634-4641 (2006). 